\title{
Comparison of Internal Adaptation of Bulk-fill and Increment-fill Resin Composite Materials
}

\author{
FS Alqudaihi $\bullet \mathrm{NB}$ Cook $\bullet \mathrm{KE} \mathrm{Diefenderfer} \bullet \mathrm{MC}$ Bottino $\bullet$ JA Platt
}

\begin{abstract}
Clinical Relevance
The filling of deep, wide cavities with bulk-fill resin composites is appealing. However, some bulk-fill resin composites result in larger internal resin-dentin gaps than observed for an incremental-fill resin composite.
\end{abstract}

\section{SUMMARY}

Objectives: To evaluate 1) the internal adaptation of a light-activated incremental-fill and bulk-fill resin-based composite (RBC) materials by measuring the gap between the restorative material and the tooth structure and 2) the aging effect on internal adaptation.

*Fatema Sabri Alqudaihi, BDS, MSD, Dental Department, Qatif Central Hospital, Qatif, Eastern Province, Saudi Arabia

N Blaine Cook, DDS, MSD, Department of Cariology, Operative Dentistry and Dental Public Health, Division of Operative Dentistry, Indiana University School of Dentistry, Indianapolis, IN, USA

Kim E Diefenderfer, DMD, MS, MS, Department of Cariology, Operative Dentistry and Dental Public Health, Division of Operative Dentistry, Indiana University School of Dentistry, Indianapolis, IN, USA

Marco C Bottino, DDS, PhD, Department of Cariology, Restorative Sciences, and Endodontics, University of Michigan School of Dentistry, Ann Arbor, MI, USA

Jeffrey A Platt, DDS, MS, Department of Biomedical and Applied Sciences, Division of Dental Biomaterials, Indiana University School of Dentistry, Indianapolis, IN, USA

*Corresponding author: PO Box 958, Qatif 31911, Saudi Arabia; e-mail: fatema.sabri@hotmail.com

DOI: $10.2341 / 17-269-\mathrm{L}$
Methods and Materials: Seventy teeth with class I cavity preparations were randomly distributed into five groups; four groups were restored with bulk-fill RBCs: Tetric EvoCeram Bulk Fill (TEC), SonicFill (SF), QuiXX Posterior Restorative (QX), and X-tra fil (XF); the fifth group was restored with incremental-fill Filtek Supreme Ultra Universal Restorative (FSU). One-half of the specimens of each group were thermocycled. Each tooth was sectioned, digital images were recorded, and the dimensions of any existing gaps were measured. Data were analyzed using analysis of variance $(\alpha=0.05)$.

Results: FSU had the smallest gap measurement values compared with the bulk-fill materials except QX and TEC $(p \leq 0.008)$. FSU had the smallest sum of all gap category values compared with the bulk-fill materials, except QX $(p \leq 0.021)$. The highest gap incidence and size values were found at the composite/adhesive interface. All aged groups had greater gap values in regard to the gap measurement and the sum of all gap categories compared with non-aged groups.

Significance: The incrementally placed material FSU had the highest internal adaptation to the cavity surface, while the four bulk-fill 
materials showed varied results. Thermocycling influenced the existing gap area magnitudes. The findings suggest that the incremental-fill technique produces better internal adaptation than the bulk-fill technique.

\section{INTRODUCTION}

Given their numerous advantages, resin-based composite (RBC) materials have been widely used in dentistry since their development in the late 1950s. Besides having esthetic properties that mimic those of natural teeth, $\mathrm{RBC}$ does not require the removal of healthy tissues to achieve retention because of the ability to bond to tooth structure using resin adhesives. As a result, RBC has several indications: direct filling of anterior and posterior caries lesions and tooth defects, esthetic bonding, and as a luting cement for indirect restorations or the bonding of orthodontic brackets. ${ }^{1}$

One reason for $\mathrm{RBC}$ direct restoration failure is postoperative pain or sensitivity. The hydrodynamic theory proposes that any change in fluid pressure and fluid movement stimulates pain receptors in the pulp that would cause postoperative pain and sensitivity following placement of resin composite restorations. ${ }^{2}$ Poor internal adaptation of a resin composite restoration will create gaps between the material and the tooth structure and allow fluid collection. This fluid will move into the dentinal tubules due to pressure changes created during mastication or temperature fluctuations, causing the patient to feel pain. ${ }^{3}$ Other possible reasons for $\mathrm{RBC}$ restoration failure are material loss resulting in gap formation and inadequate internal adaptation that can impact retention. ${ }^{3}$

Many factors, including material properties, cavity preparation design, and operative technique, influence the quality of adaptation of RBC restorations. However, polymerization shrinkage and its associated stress are two of the most adverse properties of current materials. ${ }^{3}$ Many clinical techniques, such as controlling the curing light intensity, ${ }^{4}$ indirect placement of resin restorations, ${ }^{4}$ application of a flowable resin liner, ${ }^{5}$ and incremental layering techniques, ${ }^{4}$ have been introduced to minimize the shrinkage stress and thereby reduce gap formation. However, no method has been shown to be totally effective in abating the effects of polymerization shrinkage. ${ }^{6}$

The incremental, or layering, technique is a common approach used to eliminate gap formation and achieve adequate bonding of composite to tooth structure. ${ }^{6,7}$ The technique involves placing $\mathrm{RBC}$ in multiple increments, with each increment placed to a specific thickness (2 $\mathrm{mm}$ or less) in an oblique manner, and polymerized separately. Thus, the technique allows adequate light penetration to cure the material. ${ }^{8}$ Moreover, it decreases the C-factor (the ratio of bonded surface to unbonded free surface) ${ }^{6,9}$ However, this technique has some drawbacks, such as increased chair time and inclusion of voids between the increment layers. ${ }^{6}$

Many efforts have been made to develop RBC materials that can fill larger cavities all at once, utilizing a bulk-fill technique. Unlike traditional RBCs, bulk-fill RBCs are intended to be placed in increments of $4 \mathrm{~mm}$ or greater. ${ }^{8}$ When the entire cavity can be filled in one step, this technique is easier, saves time, and results in fewer voids throughout the material.

An increased material thickness can inhibit good light penetration through the material. While the external surface may cure sufficiently, the material may not polymerize well in deeper portions. ${ }^{10}$ Over the past two decades, many manufacturers have introduced RBC materials that are claimed to have increased depth of cure and decreased polymerization shrinkage and gap formation, which would lend the materials to a bulk-fill placement technique. Recently, some of these products have gained popularity, and multiple studies indicate comparable physical and mechanical properties among bulk-fill and incremental-fill composite materials. ${ }^{10-12}$ These studies support the claim that bulk-fill materials have greater depth of cure and lower polymerizationinduced shrinkage stress than the traditional incremental-fill materials. ${ }^{13,14}$

Therefore, bulk-fill restorative products may be considered viable alternatives to the traditional incremental-fill materials in preparations with high C-factor if increased depths of cure are seen with less internal stress. Reduced polymerization stress would decrease the incidence of gap formation and result in better internal adaptation compared with traditional incrementally placed composites. ${ }^{15}$ Further investigation of the internal adaptation between the restorative material and the tooth structure is needed. The purposes of this study were to 1) quantitatively evaluate the internal adaptation among different bulk-fill RBC materials and a traditional RBC placed incrementally by measuring the gap area between the restorative material and the tooth structure and 2) evaluate the effect of aging on the internal adaptation. 
Table 1: Artificial Saliva (OHRI Recipe With Mucin and Buffer, $\mathrm{pH}=7$ )

\begin{tabular}{|lcccccc|}
\hline Material & $\mathrm{CaCl}_{2} \mathrm{H}_{2} \mathrm{O}$ & $\mathrm{KH}_{2} \mathrm{PO}_{4}$ & $\mathrm{KCl}$ & $\mathrm{NaCl}$ & Tris Buffer & Gastric Mucin \\
\hline Weight/1 L & $0.213 \mathrm{~g}$ & $0.738 \mathrm{~g}$ & $1.114 \mathrm{~g}$ & $0.381 \mathrm{~g}$ & $12 \mathrm{~g}$ & $2.20 \mathrm{~g}$ \\
\hline Abbreviation: $\mathrm{OHRl}$, Oral Health Research Institute, Indiana University School of Dentistry, Indianapolis. & & \\
\hline
\end{tabular}

\section{METHODS AND MATERIALS}

\section{Tooth Preparation}

Seventy extracted, unrestored, and caries-free human maxillary $(\mathrm{n}=13)$ and mandibular $(\mathrm{n}=57)$ molar teeth were collected and stored in $0.10 \%$ thymol (local institutional review board number 1505861672) until they were used in this in vitro study. The cusps of each tooth were flattened by grinding perpendicular to the long axis of the tooth using a 400-grit silicon carbide paper under water lubrication. This created a flat exposed dentin surface in the cusp tip areas. The enamel remained intact at the center of the occlusal surface so that all occlusal cavosurface margins of the cavity preparations were in enamel. This step was required to allow the light-curing units to be held at a repeatable distance from the occlusal margin when photo-curing each tooth. Using an ISOMET 1000 precision saw (Buehler, Lake Bluff, IL, USA) with water cooling, the root portion was removed to 1 $\mathrm{mm}$ apical to the cementoenamel junction and parallel to the flat cusp surfaces to simplify the later sectioning procedure for each specimen. The teeth were stored in artificial saliva $(\mathrm{pH}=7$; composition listed in Table 1 ) at $37^{\circ} \mathrm{C}$.

In the central fossa of each tooth, a class I cavity (Cfactor $=5.0$ ) was prepared with a round-ended straight fissure carbide bur (cylindrical, \#1158, SS White Burs Inc, Lakewood, NJ, USA) at high speed in a contraangle air-turbine hand piece with air/water spray. The teeth, together with the hand piece, were mounted on a Lathe Model 4100 (Sherline Products Inc, Vista, CA, USA) to produce standardized cavity preparation dimensions. The cavity dimensions were as follows: the buccolingual extension was $2 \mathrm{~mm}$ $( \pm 0.2 \mathrm{~mm})$, the mesiodistal extension was $6 \mathrm{~mm}( \pm 0.2$ $\mathrm{mm})$, and the occlusogingival depth was $4 \mathrm{~mm}( \pm 0.2$ $\mathrm{mm}$ ). The initial entrance of the bur was made at the mesial pit perpendicular to the long access of each tooth with the depth of $3.5 \mathrm{~mm}$, crossing the central groove (of the lower molars) and the oblique ridge (of the upper molars) whenever necessary to obtain the 6-mm mesiodistal dimension. The bur was changed after every five cavity preparations. The internal angles were rounded, and the dimensions of each preparation were validated using a Michigan-O probe under $3.0 \times$ operator loupe magnification with LED headlight illumination; any required slight cavity modifications were made manually. Preparations with cracks at the margins or observed pulpal exposure were not used in the experiment.

\section{Specimen Preparation}

The prepared teeth were randomly distributed to five experimental groups $(n=14)$; each group contained at least two upper molar teeth. Each group was restored with a different RBC system. The materials were selected from commercially available bulk-fill RBC systems, including only those that do not require veneering with an occlusal layer of conventional highviscosity composite. The materials were a traditional universal composite placed by increment-fill technique (FSU, Filtek Supreme Ultra Universal Restorative, 3M ESPE, St Paul, MN, USA) and four composite materials placed by a bulk-fill technique (TEC, Tetric EvoCeram Bulk Fill, Ivoclar Vivadent, Schaan, Liechtenstein; SF, SonicFill, Kerr, Orange, CA, USA; QX, QuiXX Posterior Restorative, Dentsply DeTrey GmbH, Konstanz, Germany; and XF, x-tra fil, Voco GmbH, Cuxhaven, Germany) (Table 2).

All prepared cavities were acid-etched and bonded using the same total-etch system (Kerr Gel Etchant and OptiBond Solo Plus, Kerr) to reduce the number of variables, as there were no manufacturer restrictions for the adhesive. Each cavity was etched with $37.5 \%$ phosphoric acid for 15 seconds and then rinsed thoroughly with copious amounts of water for 15 seconds. A moist dentin surface was maintained by blotting excess moisture from the dentin with a cotton pellet. Then two layers of the bonding agent were applied with a microbrush, using a light rubbing motion for 15 seconds for each layer. After applying a weak stream of air for three seconds to evaporate solvent, the bonding agent was light cured (DEMI LED, Kerr) with an irradiance of $1615 \mathrm{~mW} / \mathrm{cm}^{2}$ for 20 seconds. Light output was monitored using a Managing Accurate Resin Curing calibrator (MARC Resin Calibrator, BlueLight Analytics Inc, Halifax, NS, Canada). The curing light tip was fixed perpendicular to the cavity at a distance of $3.0 \mathrm{~mm}$ from the cavosurface margins using an adjustable arm clamp (MARC Resin Calibrator, BlueLight Analytics). All of the restorative materials were packed to the cavity floor and walls by using the same blunt plastic 
Table 2: Materials, Manufacturers, and Chemical Compositions of Matrix, Filler Type, and Filler Content by Weight (wt) and Volume (vol)

\begin{tabular}{|c|c|c|c|c|}
\hline Group Code/Product & $\begin{array}{c}\text { Manufacturer, Shade, } \\
\text { Part Number }\end{array}$ & Resin Matrix & Filler Composition/Size & $\begin{array}{l}\text { Filler Amount, } \\
\text { Wt } \% / \text { Vol } \%\end{array}$ \\
\hline \multicolumn{5}{|c|}{ Traditional increment-fill resin composite } \\
\hline $\begin{array}{l}\text { FSU: Filtek Supreme } \\
\text { Ultra Universal } \\
\text { Restorative (nanohybrid) }\end{array}$ & 3M ESPE, A2B, 6029A2B & $\begin{array}{l}\text { Bis-GMA, UDMA, } \\
\text { TEGDMA, Bis-EMA, } \\
\text { PEGDMA }\end{array}$ & $\begin{array}{l}\text { Silica, zirconia, zirconia/ } \\
\text { silica }(0.6-10 \mu \mathrm{m})\end{array}$ & $78.5 / 63.3$ \\
\hline \multicolumn{5}{|l|}{ Bulk-fill resin composites } \\
\hline $\begin{array}{l}\text { TEC: Tetric EvoCeram } \\
\text { Bulk Fill (nanohybrid) }\end{array}$ & $\begin{array}{l}\text { Ivoclar Vivadent, universal } \\
\text { IVA, }{ }^{a} 638244 W W\end{array}$ & $\begin{array}{l}\text { Bis-GMA, UDMA, } \\
\text { Bis-EMA }\end{array}$ & $\begin{array}{l}\text { Barium glass, ytterbium } \\
\text { trifluoride, mixed oxide, } \\
\text { prepolymerized fillers } \\
(0.04-3 \mu \mathrm{m})\end{array}$ & $76-77 / 53-54$ \\
\hline $\begin{array}{l}\text { SF: SonicFill } \\
\text { (nanohybrid) }\end{array}$ & Kerr, A2, 34922 & $\begin{array}{l}\text { Bis-GMA, Bis-EMA } \\
\text { TEGDMA, EBPDMA, } \\
\text { MPS }\end{array}$ & $\begin{array}{l}\mathrm{SiO}_{2} \text {, glass, oxide }(0.02-40 \\
\mu \mathrm{m})\end{array}$ & $67 / 83.5$ \\
\hline $\begin{array}{l}\text { QX: QuiXX Posterior } \\
\text { Restorative (hybrid) }\end{array}$ & $\begin{array}{l}\text { Dentsply DeTrey GmbH, } \\
\text { universal, } 631202\end{array}$ & $\begin{array}{l}\text { UDMA, TEGDMA, di- } \\
\text { and trimethacrylate resins, } \\
\text { carboxylic acid modified } \\
\text { dimethacrylate resin }\end{array}$ & $\begin{array}{l}\text { Strontium aluminum, } \\
\text { sodium fluoride, phosphate } \\
\text { silicate glass (NP) }\end{array}$ & $86 / 66$ \\
\hline XF: X-tra fil (hybrid) & $\begin{array}{l}\text { Voco GmbH, universal, } \\
1741\end{array}$ & $\begin{array}{l}\text { Bis-GMA, UDMA, } \\
\text { TEGDMA }\end{array}$ & $\begin{array}{l}\text { Barium boron aluminum } \\
\text { silicate glass }(0.05-10 \mu \mathrm{m})\end{array}$ & $86 / 70$ \\
\hline \multicolumn{5}{|c|}{$\begin{array}{l}\text { Abbreviations: Bis-GMA, bisphenol-A diglycidyl ether dimethacrylate; UDMA, urethane dimethacrylate; TEGDMA, triethylene glycol dimethacrylate; Bis-EMA, } \\
\text { bisphenol-A polyethylene glycol diether dimethacrylate; PEGDMA; poly(ethylene glycol) dimethacrylates; EBPDMA, ethoxylated bisphenol-A-dimethacrylate; MPS, 3- } \\
\text { trimethoxysilylpropyl methacrylate, NP, filler size not provided. } \\
\text { a Universal IVA for restorations in the "A" range (A2-A3). }\end{array}$} \\
\hline
\end{tabular}

instrument (2 Woodson composite instrument, $\mathrm{Hu}-$ Friedy Manufacturing Co LLC, Chicago, IL, USA), except for SF, which was placed using a sonicactivated hand piece. All light-curing times were according to the manufacturer instructions.

\section{FSU Group (Control)}

The cavities were restored using an oblique incremental layering technique with five wedge-shaped composite increments from the pulpal floor to the occlusal surface (each increment being not more than $2.0 \mathrm{~mm}$ thick). ${ }^{16}$ The first increment was placed against the mesial wall and the pulpal floor and then polymerized; material was then placed against the distal wall to the pulpal floor and polymerized. This procedure was repeated to place the third and fourth increments. No increment was placed at any time that would contact both the mesial and the distal walls of the preparation. The last increment filled the remaining part up to the occlusal portion of the preparation (Figure 1). Each increment was cured for 20 seconds using the same curing light and mounting device described above.

\section{TEC Group}

TEC was placed in a 4.0-mm bulk increment and then light cured for 10 seconds using the previously described method.

\section{SF Group}

SF was placed in a $4.0-\mathrm{mm}$ bulk increment and then light cured for 20 seconds using the previously described method. Material placement was facilitated by using a sonic-activated hand piece utilizing technology that lowers the material's viscosity, allowing greater flow.

\section{QX Group}

QX was placed in a 4.0-mm bulk increment and then light cured for 10 seconds using the previously described method.

\section{XF Group}

$\mathrm{XF}$ was placed in a $4.0-\mathrm{mm}$ bulk increment and then light cured for 10 seconds using the method described above.

Additional light curing from the facial and lingual surfaces was performed following the manufacturer's recommendation for $\mathrm{SF}, \mathrm{QX}$, and XF materials.

Each group was divided equally into two subgroups-aged and nonaged-with seven teeth in each. Nonaged specimens were immersed in artificial saliva at $37^{\circ} \mathrm{C}$ and stored in the dark for at least 48 hours to ensure complete material polymerization. In the aged groups, all specimens were immersed in artificial saliva at $37^{\circ} \mathrm{C}$ for 48 hours 


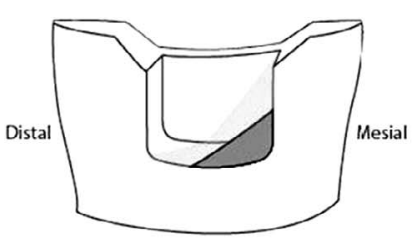

(1)

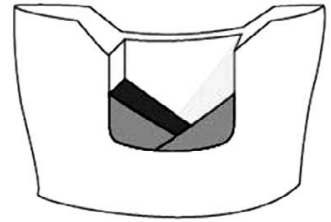

(2)

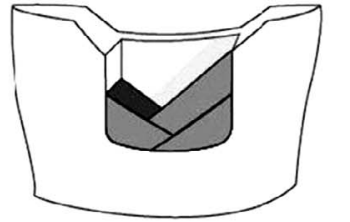

(3)

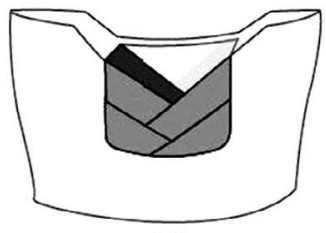

(4)

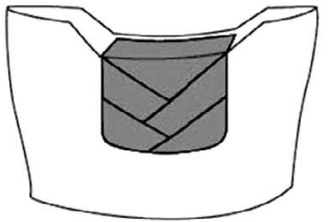

(5)
Figure 1. Cross-section view shows the successive resin composite incremental layering technique. and then thermocycled (SD Mechatronik Thermocycler, SD Mechatronik GmbH, Westerham, Germany) for 5000 cycles between $5^{\circ} \mathrm{C}$ and $55^{\circ} \mathrm{C}$, with a dwell time of 30 seconds and a transfer time of 10 seconds, which corresponded to six months of in vivo functioning. ${ }^{17}$

After the storage period, all teeth were sectioned vertically in a buccal-lingual direction using an ISOMET 1000 precision diamond blade (Buehler) under water cooling. Four cuts were made with the first cut at the mesial restoration margin, the last cut at the distal restoration margin, and two cuts in between to create three 2.0-mm-thick slices for each tooth. Internal adaptation evaluation and existing gap measurements were conducted on the interface of the inner side of the mesial (labeled as a) and distal slices (labeled as c) and both sides of the middle slice (the mesial and distal sides were labeled as $b$ and $b^{\prime}$, respectively) (Figure 2).

Each specimen was fixed on a mounting block (Struers Inc, Cleveland, OH, USA) by using sticky wax, leaving the interfaces to be measured exposed. The surfaces were ground flat with water-cooled abrasive discs (500-, 1200-, 2400-, and 4000-grit $\mathrm{Al}_{2} \mathrm{O}_{3}$ papers, MD-Fuga, Struers) and polished using a polishing cloth with a diamond suspension $(1 \mu \mathrm{m}$ DP-Suspension P; Struers). All specimens were cleaned in an ultrasonic bath and stored in a humidor using in dark, closed containers at $37^{\circ} \mathrm{C}$ until their next use.

\section{Image Recording and Analysis}

The internal adaptation, defined as the lack of any space or gap between the tooth structure and the restorative material, was evaluated along the cavity pulpal floor of each cavity preparation. Measurements were obtained from a designated area that was $1000 \mu \mathrm{m}$ long and located at the center of the cavity pulpal floor (Figure 3). The surface of each specimen was gently dried immediately before measuring by using laboratory-delicate task wipes (Kimwipes, Kimtech Science, KimberlyClark Global Sales Inc, Roswell, GA, USA). Multiple digital images were taken and saved for each of the a-, b-, b'-, and c-labeled experimental sides $(\mathrm{n}=280)$ by using a 1.3-megapixel high-resolution monochrome CCD digital camera and a light reflection microscope (Instron-Wilson-Tukon Model 2100B, Instron, Norwood, MA, USA). The images were obtained using $200 \times$ magnification; 500 $\times$ magnification was used to confirm adaptation failure through the existence of a gap. The images were stitched together by using Adobe Photoshop CS6 to create one panoramic view for each labeled specimen side.

The images were analyzed by using digital image analysis software (ImageJ, ver. 1.459r, National Institutes of Health, Bethesda, MD, USA). A region of interest was determined for each panoramic image, and measurements were obtained. The region of interest was located at the center of each pulpal floor and had dimensions of $1000 \mu \mathrm{m}$ in length and $500 \mu \mathrm{m}$ in height. Any gap at the composite/dentin interface was identified and classified into one of the following categories based on its location:

CA: gap at the composite/adhesive interface $\mathrm{AD}$ : gap at the adhesive/dentin interface 


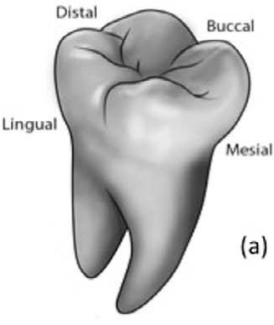

(a)

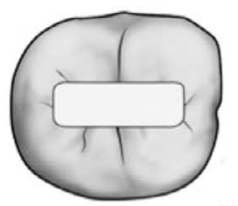

(d)
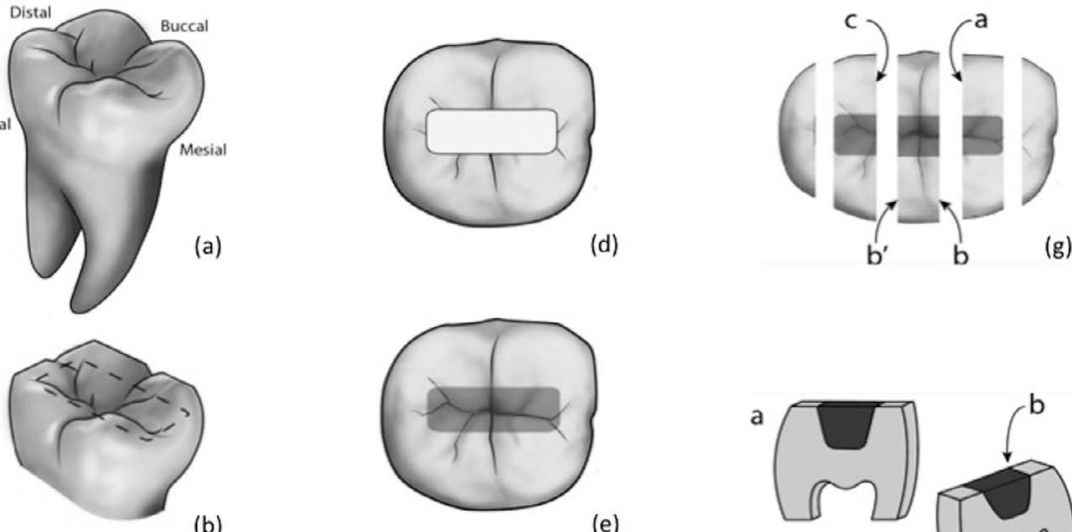

(e)

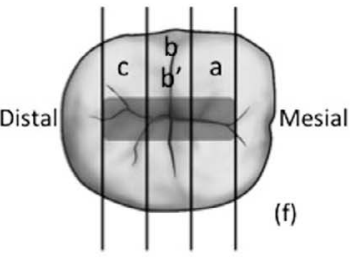

a

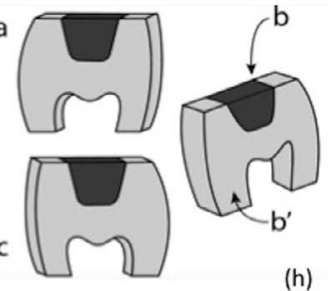

Figure 2. Specimen preparation sequence. (a): Sample lower molar. (b): Cusp tips flattening and root sectioning. (c): Cavity preparation. (d): Occlusal view for cavity dimensions: 6 $\mathrm{mm}$ for the mesiodistal extension, 2 $\mathrm{mm}$ for the buccolingual extension, and $4 \mathrm{~mm}$ for the occlusogingival depth. (e): Each experimental group filled with different resin composite material. (f): Teeth sectioning. (g): Three slices were created from each tooth, and four sides were labeled. (h): Labeling procedure for the inner side of tooth mesial slice (a), the mesial side of tooth middle slice (b), the distal side of tooth middle slice $\left(b^{\prime}\right)$, and the inner side of tooth distal slice (c).

Figure 3. The designated area that the study analysis attained was 1000 $\mu \mathrm{m}$ long and located at the center of the cavity pulpal floor.

FIG 2

(c)

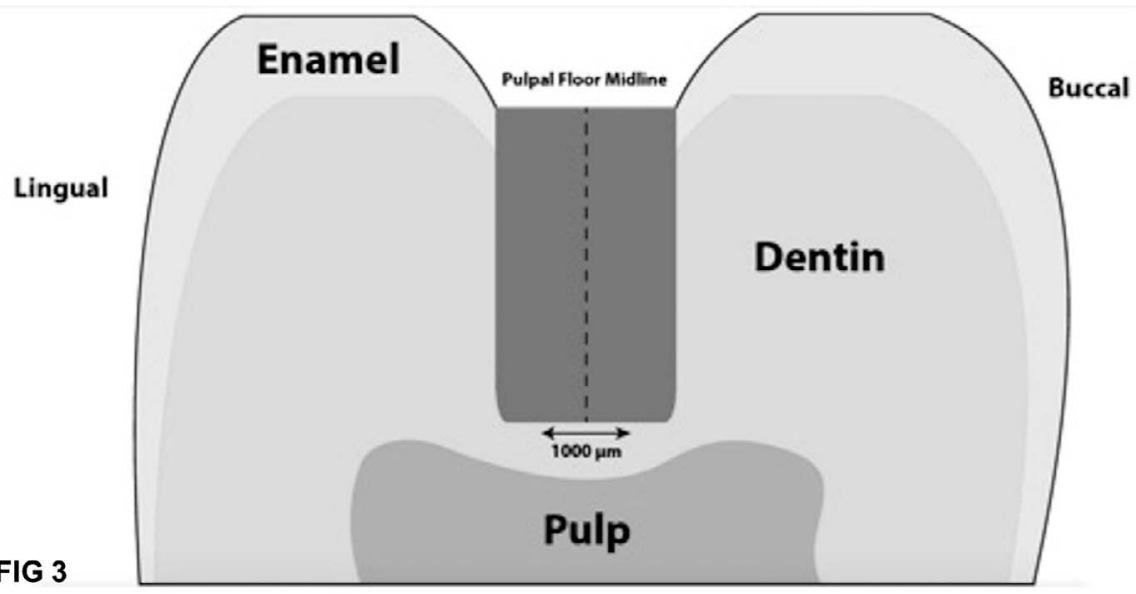

CAD: gap at the composite/adhesive/dentin interface (mixed)

Next, each gap boundary was determined, and the gap area was measured $\left(\mu \mathrm{m}^{2}\right)$ using dimensional calibration based on a high-precision-stage micrometer after threshold determination using ImageJ.

The cavity adaptation (\%) was calculated for each specimen utilizing the following equation:

Cavity Adaptation $(\%)=($ sum of adaptation length $/$ total length of cavity floor $) \times 100$.
Any cohesive failures that occurred within the composite-based material or the dentin were documented. Similarly, the occurrence of any internal voids surrounded completely by composite-based material was recorded.

A single trained operator prepared all specimens and completed all laboratory procedures. To ensure accuracy, a second trained operator also collected data and conducted measurements from the same recorded specimen images. There were similar measurements between the two evaluators mainly when gap sizes were relatively small and no differences were encountered that influenced the final results and research conclusion. 


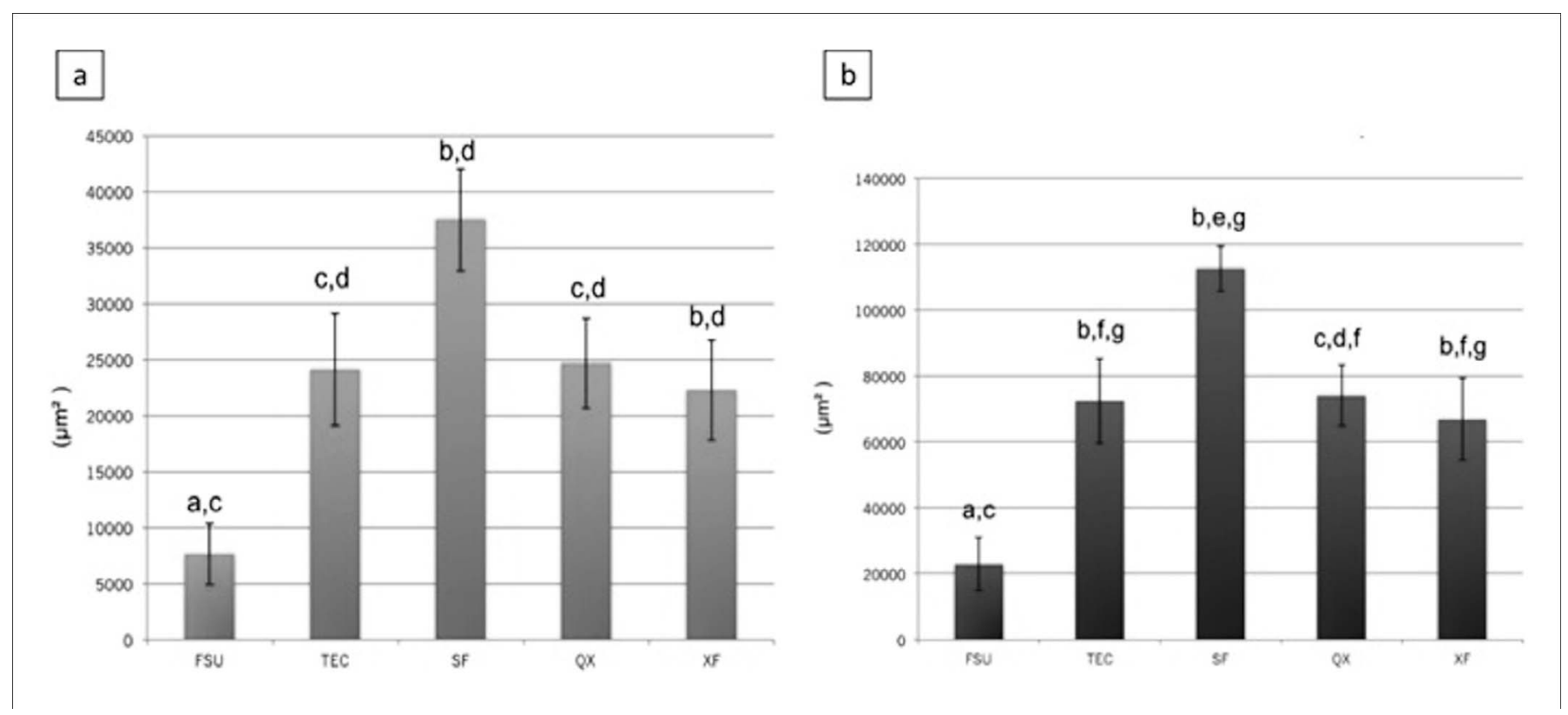

Figure 4. Mean $( \pm S D$ ) gap measurement (a) and sum of all gap categories (b) by restorative material. Groups identified with similar letters are not significantly different. Abbreviations: FSU, Filtek Supreme Ultra Universal Restorative; TEC, Tetric EvoCeram Bulk Fill; SF, SonicFill; QX, QuiXX Posterior Restorative; XF, X-tra fil.

\section{Statistical Analysis}

Summary statistics were computed for gap area measurement with and without aging of the specimens for each of the five restorative material groups and gap location categories. Mixed-model analysis of variance was performed to examine the effects of aging, restorative material groups, gap location categories, and specimen slice side with a random effect for the correlation between the slices within each tooth. An additional mixed-model analysis of variance was performed to examine the additional effect of gap location categories, with an additional random effect for correlating the gap location categories within each slice. Fisher's protected least significant difference test was used to control for multiple comparisons. Due to non-normality in the data, the dependent variables were ranked (from smallest to largest) prior to analysis.

The sum of all gap categories $(\mathrm{CA}, \mathrm{AD}$, and $\mathrm{CAD})$ and the cavity adaptation values were ranked and then used for the analysis process. There was an additional fixed and random effect in gap measurement analysis in addition to the ranking of the dependent variables of the gap measurement values. A 0.05 significance level was used for all comparisons. With a sample size of seven teeth for each aged and non-aged group, the study had $80 \%$ power to detect a mean gap ratio of 2.5 for one group compared to another, assuming twosided tests, and a coefficient of variation of 1 .

\section{RESULTS}

\section{Gap Measurements}

Mean gap area measurements are presented in graphs (Figures 4 and 5). There were significant differences in mean gap area measurement among the restorative material groups $(p=0.0333$ ). The gap area measurement for the control group FSU was significantly smaller than SF and XF $(p \leq 0.008)$. However, no significant differences were found among the other restorative material groups, as illustrated in Figure 4a. Gap area measurements differed according to location category $(p<0.0001)$. For all five material groups combined, the gap measurements for all three locations were significantly different from one another, with the smallest measurements for $\mathrm{CAD}(\mathrm{CAD}<\mathrm{AD}<\mathrm{CA}$; all $p<0.0004$ ). As shown in Figures 5 and 6 , there was a significant interaction between restorative material group and gap location $(p<0.0001)$. In the FSU group, the gap measurement for $\mathrm{CAD}$ was significantly smaller than CA $(p=0.0411)$. Gap measurements for both $\mathrm{AD}$ and $\mathrm{CAD}$ were significantly smaller than $\mathrm{CA}$ in the QX, SF, TEC, and XF groups $(p \leq 0.0024)$. CAD was significantly smaller than $\mathrm{AD}$ in the $\mathrm{QX}$ group ( $p=0010)$; however, there was no significant difference between $\mathrm{CAD}$ and $\mathrm{AD}$ gap measurements for the other four material groups (all $p \geq 0.1403$ ). The gap measurements for the aged group were significantly higher than for the nonaged group ( $p=0.0452$ ). 

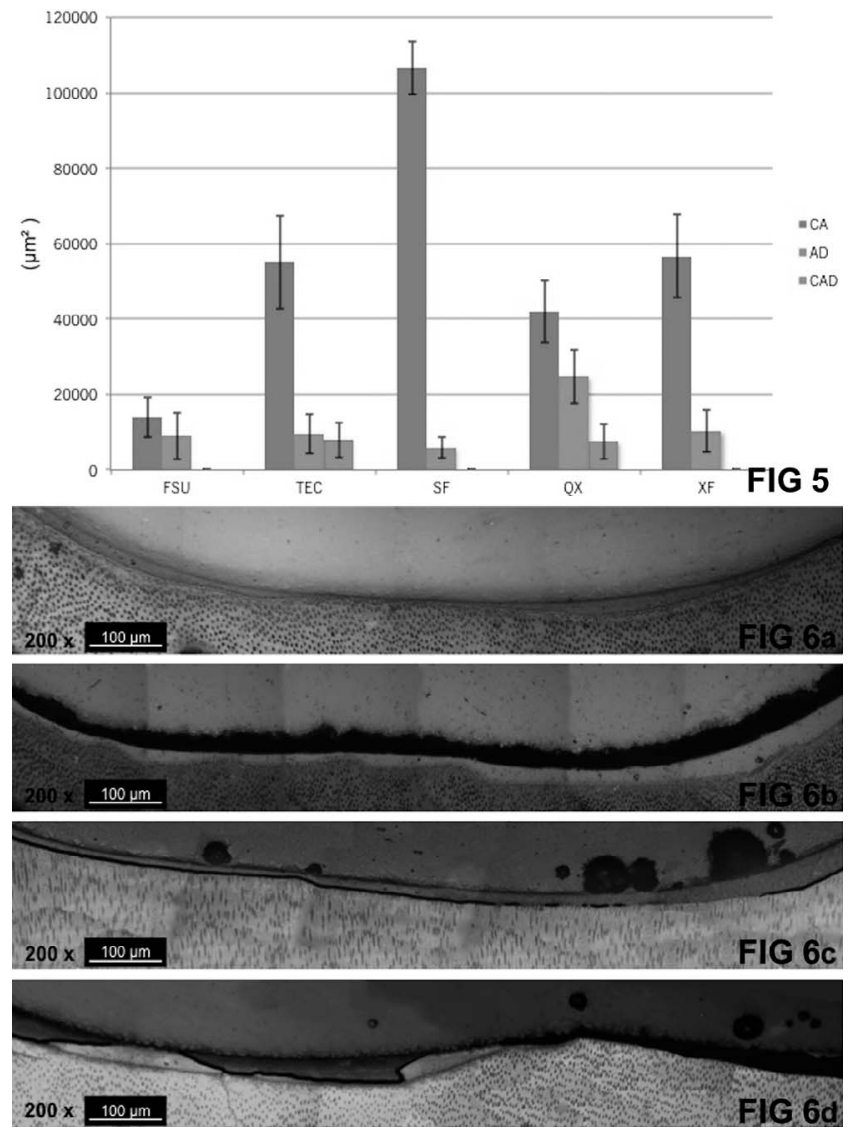

Figure 5. Mean ( $\pm S D$ ) gap measurement by gap location and restorative material.

Figure 6. Panoramic images representing different gap categories. (a): That obtained from FSU group shows no gap at all. (b): That obtained from SF group shows CA gap type. (c): That obtained from $X F$ group shows $A D$ gap type. (d): That obtained from $Q X$ group shows CAD gap type.

Overall, there were significant differences in the sum of all gap categories among the restorative material groups $(p=0.0036)$. Individual comparisons indicated that the sum of all gap categories in the control group FSU was significantly smaller than the TEC, SF, and XF groups $(p \leq 0.021)$. Similarly, the sum of all gap categories for QX was significantly smaller than SF ( $p=0.0257$ ) (see Figure $4 b)$. The sum of all gap categories for slice side a and side $\mathrm{c}$ was significantly larger than $b(p \leq 0.0155)$. The sum of all gaps for the non-aged groups was significantly higher than that for the nonaged groups $(p=0.0239)$.

In general, there were significant differences among restorative material groups $(p=0.0001)$. The control group FSU had a higher cavity adaptation than the SF, TEC, and XF groups ( $p \leq 0.019)$, and the cavity adaptation for QX and TEC was significantly higher than $\mathrm{SF}(p \leq 0.0148)$.
Table 3: Incidence of Gap Location Categories Among Different Groups of Restorative Materials

\begin{tabular}{|lrrrc|}
\hline Restorative Material Group & CA & AD & CAD & Total \\
\hline FSU & 7 & 4 & 0 & 11 \\
\hline QX & 26 & 15 & 3 & 44 \\
\hline SF & 51 & 5 & 0 & 56 \\
\hline TEC & 22 & 6 & 3 & 31 \\
\hline XF & 27 & 5 & 0 & 32 \\
\hline Abbrevatons
\end{tabular}

Abbreviations: $C A$, gap at the composite/adhesive interface; $A D$, gap at the adhesive/dentin interface; $C A D$, gap at the composite/adhesive/dentin interface (mixed)

\section{Gap Location}

Table 3 shows the incidence of gap location categories among different restorative material groups. CA gap category incidence was the highest among all the restorative groups followed by the $\mathrm{AD}$ gap category. The CAD gap category was presented only in the $\mathrm{QX}$ and TEC groups.

All cohesive failures observed in the filling material or the dentin were reported. There were 11 total cohesive failures; nine failures occurred within the composite (all groups except SF) and two within the dentin (FSU and XF). In particular, nine failures occurred in the FSU, QX, TEC, and XF nonaged groups and two in the FSU aged group. Most of the failures occurred in the XF restorative group, with a total of five failures (see Table 4).

\section{Incidence of Voids}

The incidence of any internal void that was surrounded completely by composite-based material observed in the panoramic view (from the most inferior border of the composite up to $250 \mu \mathrm{m}$ occlusally) was documented. Overall, there were significant differences in the probability of voids

\begin{tabular}{|c|c|c|c|c|}
\hline \multicolumn{5}{|c|}{$\begin{aligned} \text { Table 4: } & \text { Cohesive Failure Among Different Groups of } \\
& \text { Restorative Materials }\end{aligned}$} \\
\hline $\begin{array}{c}\text { Restorative } \\
\text { Material Group }\end{array}$ & $\begin{array}{l}\text { Aging } \\
\text { Group }\end{array}$ & Dentin & Composite & Total \\
\hline \multirow[t]{2}{*}{ FSU } & Nonaged & 0 & 1 & 3 \\
\hline & Aged & 1 & 1 & \\
\hline \multirow[t]{2}{*}{$Q X$} & Nonaged & 0 & 1 & 1 \\
\hline & Aged & 0 & 0 & \\
\hline \multirow[t]{2}{*}{ SF } & Nonaged & 0 & 0 & 0 \\
\hline & Aged & 0 & 0 & \\
\hline \multirow[t]{2}{*}{ TEC } & Nonaged & 0 & 2 & 2 \\
\hline & Aged & 0 & 0 & \\
\hline \multirow[t]{2}{*}{$\mathrm{XF}$} & Nonaged & 1 & 4 & 5 \\
\hline & Aged & 0 & 0 & \\
\hline
\end{tabular}




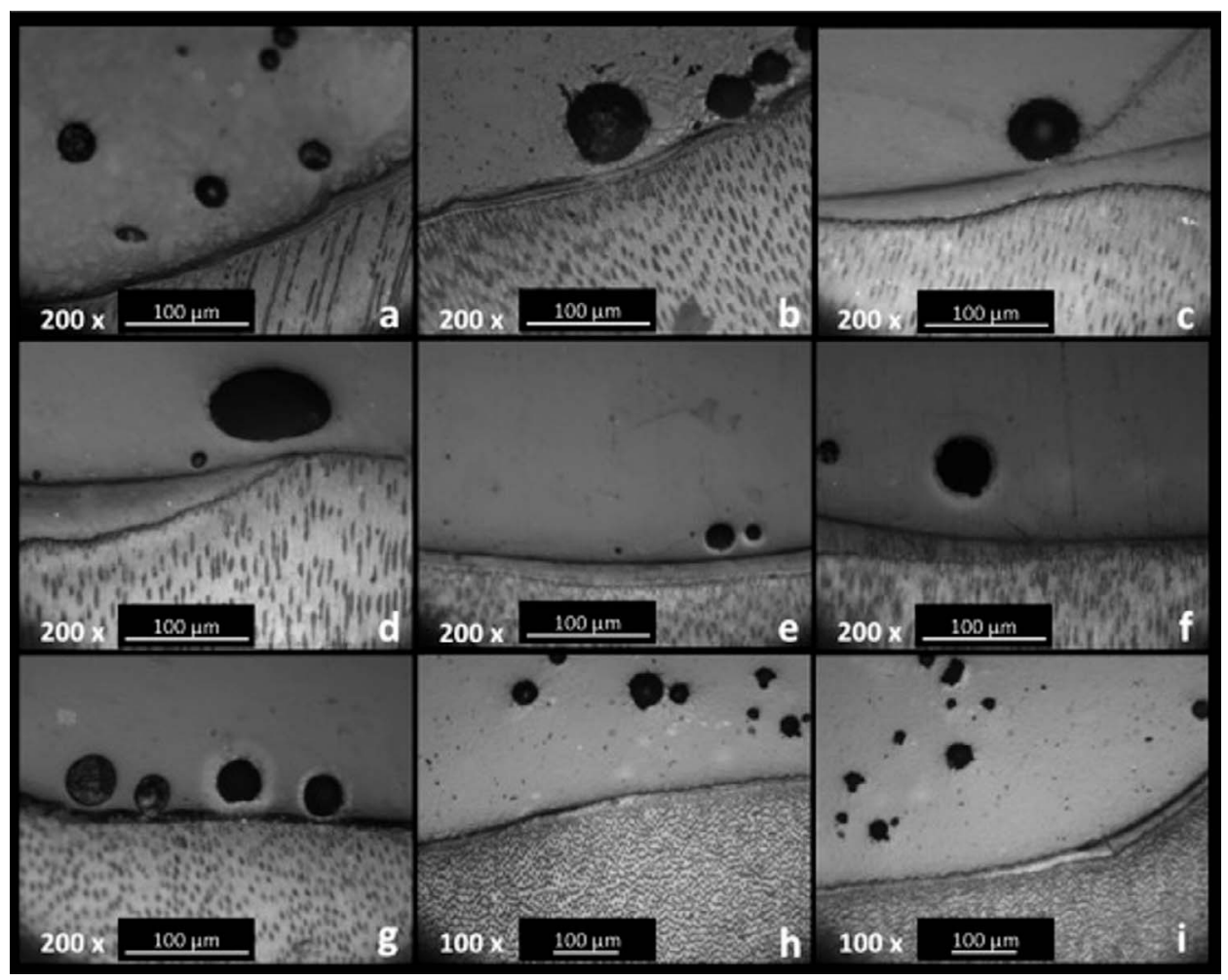

Figure 7. Internal voids. (a) and (b): Samples from XF group. (c) and (d): Samples from FSU group. (e), (f), and (g): Samples from TEC group. (h) and (i): Samples from QX group.

among the different restorative material groups $(p<0.0001)$. The SF group had a significantly lower probability of voids than FSU, QX, TEC, and XF $(p \leq 0.0024)$. QX had a significantly lower probability of voids than FSU, TEC, and XF $(p \leq 0.0287)$. However, FSU, TEC, and XF did not have significantly different probabilities of voids, as shown in Figure 7.

\section{DISCUSSION}

One of the main challenges facing RBC materials is polymerization shrinkage, which may cause the formation of gaps at both marginal and internal tooth restoration interfaces. Many authors have studied this phenomenon by measuring in vitro microleakage. However, they have generally evaluated the correlation between polymerization shrinkage and cavosurface marginal microleakage rather than internal adaptation. ${ }^{18-22}$ Furness and others ${ }^{23}$ showed fewer gap-free margins at the pulpal interface when compared with enamel or mid-dentin interfaces after restoring class I cavities with different types of bulk-fill RBC materials. Measuring the internal adaptation is more stringent than marginal microleakage since it is more challenging for the material to adapt to the deepest cavity area compared with the other interface locations.
Longitudinal clinical trials are time consuming and expensive and demand a large sample size that is challenging to standardize. Laboratory studies simulating clinical conditions remain vital as alternatives. However, there are critical factors to be considered in the experimental design to maximize the usefulness of in vitro evaluations.

First, tooth substrates vary significantly. Structural defects, such as the presence of internal crazes and enamel cracks, influence the bonding to tooth structure. Teeth with visible defects were not used in this study. Hydrated teeth have shown better bonding than dried teeth, ${ }^{24}$ so that storing and manipulating extracted teeth inconsistently might affect the results. The teeth in this study were collected under a defined storage protocol. Also, variations in anatomical features, such as cusp height and shape, can affect the final cavity preparation dimensions and C-factor, thus impacting the data. This was the motivation for using the lathe in this study that allowed the secured hand piece to be moved precisely, providing for standardized cavity dimensions.

Second, the physical sectioning method used in this study to visualize the internal adaptation of the restorations is time consuming and might damage the specimens. Nondestructive techniques, such as micro-computed tomography (micro-CT) might be 
advantageous. Several studies have used micro-CT after silver nitrate infiltration ${ }^{25-27}$ as well as sweptsource optical coherence tomography ${ }^{28}$ to evaluate the internal adaptation of RBC materials. Nevertheless, these methods present significant challenges, including being expensive, time intensive, technically challenging, and possessing a steep learning curve. Moreover, their results need to be validated by sectioning the specimens and examining them microscopically.

In the current study, the dependent variables of gap measurement, sum of all gap categories, and cavity adaptation (\%) were ranked (from smallest to largest) prior to analysis. The size of the gap area may affect the incidence and severity of postoperative pain or sensitivity following placement of the restorative material. Our study demonstrated significantly better internal adaptation with a traditional RBC material placed incrementally compared to bulk-fill RBC materials. All materials tested in bulk placement had significantly larger gap interfaces and less adaptation to the cavity floor with the exception of QX. This result is supported by a recent study that reported that an incremental-fill technique improved the adaptation of a universal composite to the cavity floor compared with a bulkfill technique. ${ }^{28}$ This outcome might be related to the reduced material volume and $\mathrm{C}$-factor of each increment, which reduced the generated contraction stresses and is in agreement with previously reported studies on the advantages of incremental filling. ${ }^{29,30}$

Factors that impact polymerization shrinkage include monomer molecular weight and concentration as well as filler size and concentration. ${ }^{31} \mathrm{FSU}$, TEC, QX, and XF all have higher filler content by weight \% than SF. These high-filler resin composites have less matrix monomer available to contribute to the polymerization process. While the space occupied by the filler particles does not contribute to the curing contraction, high filler particle loads may necessitate including low-molecular-weight monomers to ensure a proper handling viscosity. In lowviscosity materials, the motility of the monomers is active such that a greater proportion of monomers contribute to the polymerization reaction, increasing the polymerization shrinkage. ${ }^{32}$ In the present study, SF exhibited significantly larger gaps and less adaptation to the cavity compared with the other materials tested. SF also had the lowest filler content, which aids in lowering its viscosity during sonic activation. Other authors reported that SF showed excellent adaptation to cavity walls with smaller gaps and fewer voids than with conventionally lined and layered composite placement techniques. ${ }^{33,34}$

$\mathrm{AD}$ and $\mathrm{CAD}$ gap categories could have a significant clinical impact on the postoperative sensitivity due to an exposed dentinal surface. Nevertheless, poor restorative material retention and fatigue degradation could be linked to the CA gap category. The incidence and measurement of the CA gap category was significantly higher than the others, followed by $\mathrm{AD}$. Another finding of interest in the results of this experiment is that the SF group had the highest incidence of CA gap compared with the other groups. It is not clear why this occurred, even though the same bonding system was used for all the experiment groups. It might be due to the relatively higher polymerization shrinkage in SF or a poor chemical reaction between SF and OptiBond Solo Plus, even though both were from the same company (Kerr) and marketed as compatible. Further study will be necessary to evaluate SF's ability to adapt to the cavity pulpal floor using different total-etch adhesive systems.

Artificial aging techniques to examine the alterations at the interface between resin restorations and tooth cavities include 1) aging by water storage, 2 ) aging by thermocycling, and 3) aging by thermomechanical load cycling. ${ }^{35-37}$ The aging technique used in this study was selected based on a review ${ }^{17}$ demonstrating that thermocycling creates stresses similar to those seen during six months of clinical service. It is interesting to note that the aging technique used in this study was a significant factor affecting the resultant gap size measurement, although it did not affect the material adaptation to the cavity. This suggests that thermocycling worsened existing gaps but did not affect actual gap creation per se. Several previous studies showed that thermocycling or storage in water might have a slight effect on gap formation; however, thermomechanical load cycling can also efficiently cause artificial aging. ${ }^{17,38-40}$ Further investigation of the effect of the three aging techniques on gap size and bulk-fill material adaptation is recommended.

In our study, internal adaptation was evaluated in four different locations within the same tooth, as three slices obtained from each tooth allowed analysis of four different sides ( $a, b, b^{\prime}$, and c). The sum of all gap categories for slice sides a and $c$ (the mesial and distal slice sides) was significantly larger than $b$ (one of the middle slice sides) $(p \leq 0.0155)$. In other words, the gaps located closer to the center of the cavity were larger than those 
located close to the cavity sides. This implies that the shrinkage force was greater near the center of the material mass. Of the materials tested in this study, all had at least one cohesive failure within the dentin or the composite material except SF. The XF group had the highest incidence of cohesive failure, all occurring within the composite material. This might be linked directly to the material strength or degree of polymerization.

Voids within the composite material are porosities that remain unoccupied. They are generally deemed undesirable because they can impact the mechanical properties and life span of the composite. ${ }^{41}$ They can allow increased sorption in the composite material and contribute to anisotropic behavior of the composite. ${ }^{42}$ Moreover, voids can act as crack nucleation sites, contributing to crack formation and propagation and generating unpredictable behavior of the material. ${ }^{42}$ In this study, SF had a significantly lower probability of voids among the groups $(p \leq 0.0024)$, followed by QX $(p \leq 0.0287)$. The incidence of voids can result from imperfections in material processing or be related to the viscosity of the material during placement and the placement technique. A resin composite material with a high viscosity is more likely to generate voids than a lowviscosity material. As the composite fillers can have void spaces from the manufacturing process, it is challenging for a high-viscosity matrix to penetrate them, especially when the fillers are packed tightly together. ${ }^{43}$ The current study supports this idea, as it shows fewer voids associated with SF, which had a low viscosity during placement. Furthermore, it has been suggested that using an incremental-fill placement technique may result in more voids due the nature of the layering procedure, which allows air to trap between layers. ${ }^{44}$ In our study, however, although FSU (the incrementally placed group) showed a statistically higher incidence of voids compared with SF and QX, there was no statistically significant difference between FSU and the other two bulk-fill groups (TEC and XF).

\section{CONCLUSIONS}

Based on the results of the present study, an incrementally placed resin composite material (FSU) showed less gap formation and better internal adaptation to the cavity floor than the bulk-fill restorative materials. Therefore, an incremental-fill technique seems to have significant advantages in internal adaptation over a bulk-fill technique. The four materials placed using a bulk-fill technique showed various behaviors and results. Moreover, thermocycling influenced the existing gap quantities, but did not play a role in the material adaptation to the cavity.

\section{Acknowledgements}

The authors would like to thank Dr Anderson Hara and Dr Tien-Min Gabriel Chu for their assistance and guidance with the research project. Also, we thank Clif Duhn for his laboratory support.

\section{Regulatory Statement}

This study was conducted in accordance with all the provisions of the local human subjects oversight committee guidelines and policies of Indiana University. The approval code for this study is 1505861672 .

\section{Conflict of Interest}

The authors of this article certify that they have no proprietary, financial, or other personal interest of any nature or kind in any product, service, and/or company that is presented in this article.

(Accepted 30 October 2017)

\section{REFERENCES}

1. Schneider LFJ, Cavalcante LM, \& Silikas N (2010) Shrinkage stresses generated during resin-composite applications: A review Journal of Dental Biomechanics 2010(2010) 131630.

2. Pashley DH (1996) Dynamics of the pulpo-dentin complex Critical Reviews in Oral Biology and Medicine 7(2) 104-133.

3. Lutz, F, Krejci I, \& Barbakow F (1991) Quality and durability of marginal adaptation in bonded composite restorations Dental Materials 7(2) 107-113.

4. Lee MR, Cho BH, Son HH, Um CM, \& Lee IB (2007) Influence of cavity dimension and restoration methods on the cusp deflection of premolars in composite restoration Dental Materials 23(3) 288-295.

5. Alomari QD, Reinhardt JW, \& Boyera DB (2001) Effect of liners on cusp deflection and gap formation in composite restorations Operative Dentistry 26(4) 406-411.

6. Park J, Chang J, Ferracane J, \& Lee IB (2008) How should composite be layered to reduce shrinkage stress: Incremental or bulk filling? Dental Materials 24(11) 1501-1505.

7. Rullmann I, Schattenberg A, Marx M, Willershausen B, \& Ernst CP (2011) Photoelastic determination of polymerization shrinkage stress in low-shrinkage resin composites Schweizer Monatsschrift für Zahnmedizin 122(4) 294-299.

8. Tiba A, Zeller GG, Estrich CG, \& Hong A (2013) A laboratory evaluation of bulk-fill versus traditional multiincrement-fill resin-based composites Journal of the American Dental Association 144(10) 1182-1183.

9. Karthick K, Sivakumar K, Geetha Priya PR, \& Shankar S (2011) Polymerization shrinkage of composites-A review 
Journal of Indian Academy of Dental Specialists 2(2) 32-36.

10. Campodonico CE, Tantbirojn D, Olin PS, \& Versluis A (2011) Cuspal deflection and depth of cure in resin-based composite restorations filled by using bulk, incremental and transtooth-illumination techniques Journal of the American Dental Association 142(10) 1176-1182.

11. Czasch P, \& Ilie N (2013) In vitro comparison of mechanical properties and degree of cure of bulk fill composites Clinical Oral Investigations 17(1) 227-235.

12. Moorthy A, Hogg CH, Dowling AH, Grufferty BF, Benetti AR, \& Fleming GJP (2012) Cuspal deflection and microleakage in premolar teeth restored with bulk-fill flowable resin-based composite base materials Journal of Dentistry 40(6) $500-505$.

13. Dentsply International. Surefil ${ }^{\circledR} \operatorname{SDR}^{\mathrm{TM}}$ flow product brochure; Retrieved online August 22, 2013 from: http:// www.surefilsdrflow.com/sites/default/files/SureFil_ Brochure.pdf

14. Kerr. SonicFill Sonic-Activated, Bulk Fill Composite System product brochure; Retrieved online January 21, 2014 from: http://www.kerrdental.com/cms-filesystem-action? file $=$ /kerrdental-products-brochure/sonicfillsalessheet.pdf

15. Bechtold J, Dos Santos PJ, Anido-Anido A, Di Hipólito V, Alonso RC, \& D'Alpino PH (2012) Hardness, polymerization depth, and internal adaptation of class II silorane composite restorations as a function of polymerization protocol European Journal of Dentistry 6(2) $133-140$.

16. Deliperi S, \& Bardwell DN (2002) An alternative method to reduce polymerization shrinkage in direct posterior composite restorations Journal of the American Dental Association 133(10) 1387-1398.

17. De Munck JD, Van Landuyt K, Peumans M, Poitevin A, Lambrechts P, Braem M, \& Van Meerbeek B (2005) A critical review of the durability of adhesion to tooth tissue: Methods and results Journal of Dental Research 84(2) 118-132.

18. Rosin M, Urban AD, Gärtner C, Bernhardt O, Splieth C, \& Meyer G (2002) Polymerization shrinkage-strain and microleakage in dentin-bordered cavities of chemically and light-cured restorative materials Dental Materials 18(7) $521-528$.

19. Braga RR, Ferracane JL, \& Condon JR (2002) Polymerization contraction stress in dual-cure cements and its effect on interfacial integrity of bonded inlays Journal of Dentistry 30(7) 333-340.

20. Peutzfeldt A, \& Asmussen E (2004) Determinants of in vitro gap formation of resin composites Journal of Dentistry 32(2) 109-115.

21. Calheiros FC, Sadek FT, Braga RR, \& Cardoso PEC (2004) Polymerization contraction stress of low-shrinkage composites and its correlation with microleakage in class V restorations Journal of Dentistry 32(5) 407-412.

22. Gerdolle DA, Mortier E, \& Droz D (2008) Microleakage and polymerization shrinkage of various polymer restorative materials Journal of Dentistry for Children 75(2) $125-133$
23. Furness A, Tadros MY, Looney SW, \& Rueggeberg FA (2014) Effect of bulk/incremental fill on internal gap formation of bulk-fill composites Journal of Dentistry 42(4) 439-449.

24. Suliman AH, Boyer DB, \& Lakes RS (1994) Polymerization shrinkage of composite resins: Comparison with tooth deformation Journal of Prosthetic Dentistry 71(1) 7-12.

25. Kim HJ, \& Park SH (2014) Measurement of the internal adaptation of resin composites using micro-CT and its correlation with polymerization shrinkage Operative Dentistry 39(2) e57-e70.

26. Kwon OH, \& Park SH (2012) Evaluation of internal adaptation of dental adhesive restorations using microCT Restorative Dentistry and Endodontics 37(1) 41-49.

27. Han SH, \& Park SH (2014) Micro-CT evaluation of internal adaptation in resin fillings with different dentin adhesives Restorative Dentistry and Endodontics 39(1) 24-31.

28. Bakhsh TA, Sadr A, Shimada Y, Mandurah MM, Hariri I, Alsayed EZ, Tagami J, \& Sumi Y (2013) Concurrent evaluation of composite internal adaptation and bond strength in a class-I cavity Journal of Dentistry 41(1) 60-70.

29. Chikawa H, Norimichi INAI, Eitetsu CHO, Kishikawa R, Otsuki M, Foxton RM, \& Tagami J (2006) Effect of incremental filling technique on adhesion of light-cured resin composite to cavity floor Dental Materials Journal 25(3) 503-508.

30. Nikolaenko SA, Lohbauer U, Roggendorf M, Petschelt A, Dasch W, \& Frankenberger R (2004) Influence of c-factor and layering technique on microtensile bond strength to dentin Dental Materials 20(6) 579-585.

31. Braga RR, Ballester RY, \& Ferracane JL (2005) Factors involved in the development of polymerization shrinkage stress in resin-composites: A systematic review Dental Materials 21(10) 962-970.

32. Davidson CL, \& Feilzer AJ (1997) Polymerization shrinkage and polymerization shrinkage stress in polymerbased restoratives Journal of Dentistry 25(6) 435-440.

33. Ferracane JL (2011) Resin composite-State of the art Dental Materials 27(1) 29-38.

34. Munoz-Viveros C, Yazici AR, Agarwal I, \& CampilloFunollet M (2012) Microleakage in class II preparations restored with the SonicFill System In $A A D R / C A D R$ Annual Meeting \& Exhibition 29-38.

35. Shono Y, Terashita M, Shimada J, Kozono Y, Carvalho RM, Russell CM, \& Pashley DH (1999) Durability of resin-dentin bonds Journal of Adhesive Dentistry 1(3) 211-218.

36. Leloup G, D'hoore W, Bouter D, Degrange M, \& Vreven J (2001) Concise review biomaterials \& bioengineering: Meta-analytical review of factors involved in dentin adherence Journal of Dental Research 80(7) 1605-1614.

37. Nikaido T, Kunzelmann KH, Chen H, Ogata M, Harada N, Yamaguchi S, Cox CF, Hickel R, \& Tagami J (2002) Evaluation of thermal cycling and mechanical loading on 
bond strength of a self-etching primer system to dentin Dental Materials 18(3) 269-275.

38. Heintze S, Forjanic M, \& Cavalleri A (2008) Microleakage of class II restorations with different tracers-comparison with SEM quantitative analysis Journal of Adhesive Dentistry 10(4) 259-267.

39. Abdalla AI, \& Davidson CL (1996) Effect of mechanical load cycling on the marginal integrity of adhesive class I resin composite restorations Journal of Dentistry 24(1-2) 87-90.

40. Mitsui FHO, Bedran-de-Castro AKB, Ritter AV, Cardoso PEC, \& Pimenta LAF (2003) Influence of load cycling on marginal microleakage with two self-etching and two onebottle dentin adhesive systems in dentin Journal of Adhesive Dentistry 5(3) 209-216.
41. ASTM International (2009) ASTM D2734-09 Standard test methods for void content of reinforced plastics, West Conshohocken, PA; ASTM International; Retrieved online February 21, 2014 from: http://www.astm.org

42. Hull D, \& Clyne TW (1996) An Introduction to Composite Materials Cambridge University Press, Cambridge.

43. Shakya N, Roux JA, \& Jeswani AL (2013) Effect of resin viscosity in fiber reinforcement compaction in resin injection pultrusion process Applied Composite Materials 20(6) 1173-1193.

44. Park J, Chang J, Ferracane J, \& Lee IB (2008) How should composite be layered to reduce shrinkage stress: Incremental or bulk filling? Dental Materials 24(11) 1501-1505. 